\title{
Correlation Processing of a Cuboid of the Infrared Images Received from Unmanned Aerial Vehicles. Part 1. Modeling and Processing of Infrared Signatures of Technogenic Objects in the Course of Daily Change of Temperatures
}

Igor' N. Ishchuk*a,b, Yury Y. Gromov ${ }^{\mathrm{c}}$, Konstantin V. Postnova, Yevgeniy A. Stepanov a and Valery N. Tyapkin ${ }^{\mathrm{b}}$ ${ }^{a}$ Military Training and Research Center of the Air Force «Air Force Academy ft. Professor N. E. Zhukovsky and Y.A. Gagarin» 54A Starykh Bol'shevikov Str., Voronezh ,394064, Russia ${ }^{b}$ Siberian Federal University 79 Svobodny, Krasnoyarsk, 660041, Russia 'Tambov State Technical University 106 Sovetskaya Str., Tambov, 392000, Russia

Received 01.12.2015, received in revised form 24.12.2015, accepted 17.01.2016

In article the technology of formation and correlation processing of a cuboid of IR images of technogenic objects is considered during daily supervision. A general statement of the problem of unsteady heat transfer in rectangular area of the material, anisotropic concerning heat conductivity approximating technogenic object is presented. Results of processing are given by correlation algorithm of dynamic IR signatures received as a result of numerical modeling.

Keywords: thermal tomogram, thermal radiation, thermal contrast, dynamic IR signature, heat conductivity of materials, thermodynamic temperature, image IR cuboids, thermal exchange, hidden objects.

Citation: Ishchuk I.N., Gromov Y.Y., Postnov K.V., Stepanov Y.A., Tyapkin V.N. Correlation processing of a cuboid of the infrared images received from unmanned aerial vehicles. Part 1. Modeling and processing of infrared signatures of technogenic objects in the course of daily change of temperatures, J. Sib. Fed. Univ. Eng. technol., 2016, 9(3), 310-318. DOI: 10.17516/1999-494X-2016-9-3-310-318.

(c) Siberian Federal University. All rights reserved

* Corresponding author E-mail address: boerby@rambler.ru 


\title{
Корреляционная обработка кубоида
}

инфракрасных изображений, получаемых

с беспилотных летательных аппаратов.

Часть 1. Моделирование и обработка

инфракрасных сигнатур техногенных объектов

в процессе суточного изменения температур

\author{
И.Н. Ищук ${ }^{\mathrm{a}, \tilde{\sigma}}$ Ю.Ю. Громов ${ }^{\mathrm{B}}$, \\ К.В. Постнов ${ }^{\text {a }}$, Е.А. Степанов ${ }^{\text {a }}$, В.Н. Тяпкин ${ }^{6}$ \\ ${ }^{a}$ ВУНЦ ВВС «ВВА им. проф. Н.Е. Жуковского и Ю.А. Гагарина» \\ Россия, 371600, Воронеж, ул. Старых Большевиков, 54а \\ ${ }^{6}$ Сибирский федеральный университет \\ Россия, 660041, Красноярск, пр. Свободный, 79 \\ ${ }^{6}$ Тамбовский государственный технический университет \\ Россия, 392000, Тамбов, ул. Советская, 106
}

В статье рассматривается технология формирования и корреляционной обработки кубоида ИК-изображений техногенных объектов в ходе суточных наблюдений. Представлена общая постановка задачи нестационарного теплообмена в прямоугольной области анизотропного в отношении теплопроводности материала, аппроксимирующего техногенный объект. Приведены результаты обработки корреляиионным алгоритмом динамических ИК-сигнатур, полученных в результате численного моделирования.

Ключевые слова: тепловая томограмма, тепловое излучение, тепловой контраст, динамические ИК-сигнатуры, теплопроводность материалов, термодинамическая температура, кубоид ИК-изображения, тепловой обмен, скрытые объекты.

\section{Введение}

Анализируя существующие методы контроля техногенных объектов на основе применения комплексов с беспилотными летательными аппаратами (БЛА), необходимо сделать вывод, что их обнаружение на фоне местности производится по тепловому излучению - тепловым контрастам. Объекты различают по размерам, конфигурации поверхности, материалам, покрытию, возможности их маскировки, скорости и направлению движения, по среде, по их термодинамическим температурам и другим параметрам. Фоны, на которых находятся объекты, каталогизируют с учетом их параметров: средних значений и среднеквадратичных отклонений термодинамических температур, обусловленных сложной изменчивостью климатических условий; спектральных характеристик излучения; пространственных неоднородностей физических структур. Исходя из этого, перечень основных параметров объектов, фонов и связанных с ними изменений значений тепловых контрастов очень велик. Поэтому проведение исследований влияния всех вышеперечисленных параметров на величины тепловых контрастов, с учетом различного сочетания множества объектов и множества фонов, снимаемых тепловизионной аппаратурой БЛА, не представляется возможным [1,2]. 


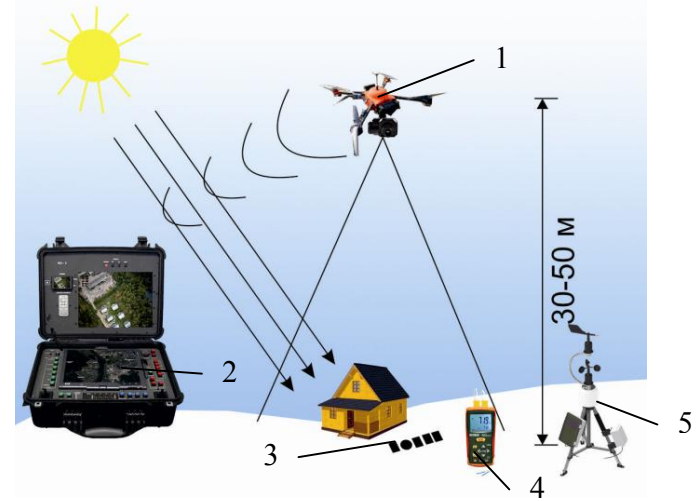

Рис. 1. Схема определения теплофизических параметров техногенных объектов: 1 - БЛА квадрокоптерного типа с тепловизором на борту; 2 - станция управления БЛА; 3 - модель техногенного объекта (техники) и эталонных материалов; 4 - термометр; 5 - мобильная метеостанция

С другой стороны, данные практических исследований дистанционного измерения температур, начатых с середины прошлого века, в начале в ИК-диапазоне длин волн, а затем и в радиодиапазоне показывают, что величины тепловых контрастов в большей степени определяются теплофизическими параметрами материалов, из которых изготовлены объекты, их размерами, свойствами подстилающей поверхности и метеорологическими условиями [3-6]. На рис. 1 приведена схема проведения дистанционных измерений теплофизических параметров техногенных объектов путем получения их ИК-сигнатур. Съемка экспозиции техногенного объекта производится с заданной периодичностью в течение суток, что позволяет сформировать кубоид ИК-изображений $[1,2]$.

На основании этого исследование закономерностей динамического изменения тепловых контрастов (температурных контрастов) материалов, связанных с практически не изменяющимися теплофизическими параметрами объектов и фонов, является актуальной задачей. Решение указанной задачи позволяет в ходе дистанционной оценки теплофизических параметров характеризовать все объекты по своим, присущим только им, параметрам с проведением их типизации [7].

\section{Постановка задачи}

Рассмотрим процесс нестационарного теплообмена в прямоугольной области анизотропного в отношении теплопроводности материала. Если в условиях однозначности решения в качестве граничных условий рассматривать условия первого рода, то математическая модель этого процесса имеет вид следующей краевой задачи:

$$
C(T) \frac{\partial T}{\partial \tau}=\frac{\partial}{\partial x}\left(\lambda_{11}(T) \frac{\partial T}{\partial x}\right)+\frac{\partial}{\partial y}\left(\lambda_{22}(T) \frac{\partial T}{\partial y}\right)+2 \frac{\partial}{\partial x}\left(\lambda_{12}(T) \frac{\partial T}{\partial y}\right)+S(x, y, \tau)
$$

где $x \in[0, a] ; y \in[0, b] ; \tau \in\left[0, \tau_{m}\right]-$ координаты и время, $C=\rho \cdot c ; \rho-$ плотность; $c$ - теплоемкость; $T$ - температура; $\lambda_{11}(T), \lambda_{22}(T), \lambda_{12}(T)$ - компоненты тензора теплопроводности; 


$$
\begin{aligned}
& T(x, y, 0)=\varphi_{0}(x, y), \\
& T\left(x_{\Gamma}, y_{\Gamma}, \tau\right)=\varphi_{\Gamma}\left(x_{\Gamma}, y_{\Gamma}, \tau\right), x_{\Gamma}=\{0 ; a\}, y_{\Gamma}=\{0 ; b\}, \tau \in\left[0, \tau_{m}\right],
\end{aligned}
$$

где $\varphi_{0}(x, y), \varphi_{\Gamma}\left(x_{\Gamma}, y_{\Gamma}, \tau\right)$ и $S(x, y, \tau)$ - известные функции начального распределения поля температур, граничных условий и функции объемного источника тепла.

Теплофизические параметры зададим в виде функции $\lambda_{11}(T), \lambda_{22}(T), \lambda_{12}(T)$ и $C(T)$, зависящих от мгновенных значениях температур в определенных $n$ точках прямоугольной области $G=[0 ; a] \times[0 ; b]$, при $T\left(x_{i}, y_{i}, \tau\right)=f\left(x_{i}, y_{i}, \tau\right), i=1,2, \ldots, n$ и известных функциях $S(x, y, \tau)$, $\varphi_{0}(x, y), \varphi_{\Gamma}\left(x_{\Gamma}, y_{\Gamma}, \tau_{\Gamma}\right)$.

Для упрощения теплофизические параметры $\lambda_{11}(T), \lambda_{22}(T), \lambda_{12}(T)$ и $C(T)$ зададим в параметризованном виде:

$$
\begin{gathered}
\lambda_{11}(T)=\sum_{k=1}^{m+3} \lambda_{11}^{(k)} L_{k}(T), \lambda_{22}(T)=\sum_{k=1}^{m+3} \lambda_{22}^{(k)} L_{k}(T), \lambda_{12}(T)=\sum_{k=1}^{m+3} \lambda_{12}^{(k)} L_{k}(T) \\
C(T)=\sum_{k=1}^{m+3} C^{(k)} L_{k}(T)
\end{gathered}
$$

где $L_{k}(T)$ - последовательность интерполяционных полиномов Лагранжа; $T \in\left[T_{\min }, T_{\max }\right]$; $m$ - число участков разбиения области определения функций при осуществлении их сплайн-аппроксимации; $\quad \lambda_{11}=\left(\lambda_{11}^{(k)}, k=1,2 \ldots,(m+3)\right), \quad \lambda_{22}=\left(\lambda_{22}^{(k)}, k=1,2 \ldots,(m+3)\right)$, $\lambda_{12}=\left(\lambda_{12}^{(k)}, k=1,2 \ldots,(m+3)\right), C=\left(C^{(k)}, k=1,2 \ldots,(m+3)\right)$ - векторы значений параметров интерполяционных полиномов.

Кроме того, упростим

$$
\begin{gathered}
\frac{\partial}{\partial x}\left(\lambda(T) \frac{\partial T}{\partial x}\right)+\frac{\partial}{\partial y}\left(\lambda(T) \frac{\partial T}{\partial y}\right)=\lambda(T) \frac{\partial^{2} T}{\partial x^{2}}+\frac{\partial \lambda(T)}{\partial T}\left(\frac{\partial T}{\partial x}\right)^{2}+ \\
+\lambda(T) \frac{\partial^{2} T}{\partial y^{2}}+\frac{\partial \lambda(T)}{\partial T}\left(\frac{\partial T}{\partial y}\right)^{2}=\lambda(T)\left(\frac{\partial^{2} T}{\partial x^{2}}+\frac{\partial^{2} T}{\partial y^{2}}\right) \text { при } \frac{\partial \lambda(T)}{\partial T} \approx 0 .
\end{gathered}
$$

Численную оценку параметров техногенного объекта будем производить путем нахождения максимумов коэффициента взаимной корреляции между данными дистанционноизмеренных динамических значений температур и рассчитанных на основе соответствующей математической модели «эталонного» материала. В [8] аналогичный подход рассматривается в качестве метода распознавания образов, известного как корреляционное сопоставление поиска эквивалентов объекта-эталона, представленного в виде вектора, матрицы или массива. Расчет коэффициента взаимной корреляции будем производить по всему растру кубоида ИКизображений в соответствии с выражением

$$
\mathrm{J}_{\mathrm{p}, \mathrm{q}}=\frac{1}{K} \cdot \frac{\sum_{k=0}^{K}\left(\Phi[k]_{p,{ }^{*}{ }^{*}}-\bar{\Phi}[k]_{p,{ }^{*}{ }^{*}}\right) \cdot\left(\Phi[k]_{p, q}-\bar{\Phi}[k]_{p, q}\right)}{\sigma_{p,{ }^{*}{ }^{*}} \cdot \sigma_{p, q}}
$$

где $p \in \overline{0, P}, q \in \overline{0, Q}, k \in \overline{0, K}$, где $P \times Q$ - растр кубоида ИК-изображений, $\bar{\Phi}$ - математическое ожидание яркости, $\sigma-$ среднеквадратичное отклонение яркости, $p,{ }^{*}{ }^{*}{ }^{*}$-координаты отсчетов 
эталонов, $K$ - количество отсчетов во времени, $\Phi$ - относительное значение яркости $\Phi \in[0 ; 1]$, которое получают из системы уравнений

$$
\left\{\begin{array}{l}
T_{r}=\varepsilon T^{4}+(1-\varepsilon) T_{A}^{4} \\
T=\Phi\left(T_{\max }-T_{\min }\right)+T_{\min }, T_{\min } \leq T \leq T_{\max },
\end{array}\right.
$$

где $T_{A}$ - температура атмосферы; $T_{r}$ - радиационная температура; $\varepsilon-$ коэффициент излучательной способности; $T_{\min }$ и $T_{\max }-$ предельные значения температур, измеренных тепловизионным приемником.

На основании численного решения прямой задачи (1) - (4) задачу сегментации кубоида ИК-изображений сформулируем следующим образом. На каждой итерации оценки $\mathbf{J}$ по $s$-эталонам рассматриваемый пиксел кубоида ИК-изображений добавляется в соответствующий сегмент, для которого $\mathbf{J}$ имеет значение больше заданного порога. В противном случае пиксель считается сильно отличающимся от всех текущих областей и создаётся новая область - область неопределенности.

\section{Описание модели}

С использованием среды трехмерного теплового моделирования на основании (1) - (3) были построены модели техногенных объектов (объект техники, здание и бункер) и эталонных материалов, покрытых черной краской, на фоне антропогенных ландшафтов (рис. 2). В ходе проведения численного моделирования динамики изменения возникающих радиационных контрастов в среде теплового моделирования были использованы данные метеорологических наблюдений погоды, зафиксированные в интервале времени с 06:10 до 08:50 следующих суток в точке с координатами 51.61 с.ш. 39.137 в.д. на высоте 98 м над уровнем моря.

Результаты расчетов суточного изменения термодинамических температур наблюдаемых на поверхностях объектов и материалов представлены на рис. 3.

В ходе численного моделирования в соответствии со схемой определения теплофизических параметров (рис. 1) было получено по 27 ИК-сигнатур исследуемых техногенных объектов с интервалом в 1 ч, некоторые из них изображены на рис. 4.

\section{Алгоритм сегментации изображения}

Для сегментации кубоида ИК-изображений и выделения областей по схожим параметрами обработку полученных данных необходимо производить в соответствии со следующим алгоритмом:

Шаг 1. Считывание динамических ИК-изображений и формирование кубоида ИКизображений [1]. Задание координат центра эталонных объектов и формирование векторов динамических значений изменения яркости $\Phi_{p,{ }^{*} q^{*}}$.

Шаг 2. Расчет векторов численных значений математического ожидания $\bar{\Phi}_{p,{ }^{*}{ }^{*}}$ и СКО $\sigma_{p, i}^{*}{ }^{*}$ изменения яркости на интервале времени наблюдения для каждого эталонного материала.

Шаг 3. Расчет коэффициента корреляции между каждым пикселем растра кубоида ИКизображений и вектором динамических значений изменения яркостей эталонных объектов $\Phi_{p, q^{*}}$ с формированием массива невязок $\mathbf{J}_{\mathbf{P}, \mathbf{Q}}$ в соответствии с выражением (4). 


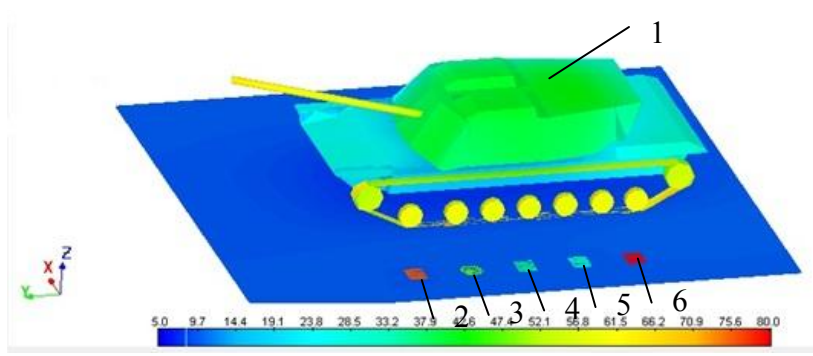

a)

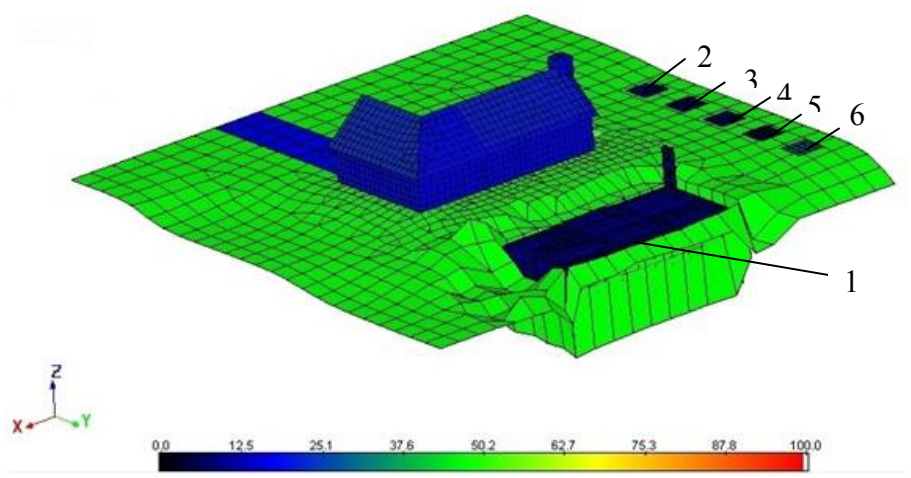

б)

Рис. 2. Тепловая модель техногенных объектов: а - объект техники; б - здание и бункер. Обозначения и параметры материалов: 1 - объект исследования (техника, скрытый объект); 2 - плита из пенопласта $(\lambda=0,04 \mathrm{BT} / \mathrm{M} \cdot \mathrm{K}, \mathrm{c}=1400$ Дж/кг $\cdot \mathrm{K}) ; 3$ - стальной лист $(\lambda=58 \mathrm{BT} / \mathrm{M} \cdot \mathrm{K}, \mathrm{c}=482$ Дж/Кг $\cdot \mathrm{K}) ; 4$ - бетонная плита $(\lambda=1,28 \mathrm{BT} / \mathrm{M} \cdot \mathrm{K}, \mathrm{c}=880$ Дж/кг $\cdot \mathrm{K}) ; 5$ - деревянная плита (сосна) $(\lambda=0,18 \mathrm{BT} / \mathrm{M} \cdot \mathrm{K}, \mathrm{c}=2300$ Дж/кг $\mathrm{K}) ; 6-$ плита из красного полнотелого кирпича $(\lambda=0,67 \mathrm{BT} / \mathrm{M} \cdot \mathrm{K}, \mathrm{c}=860$ Дж/кг·К)

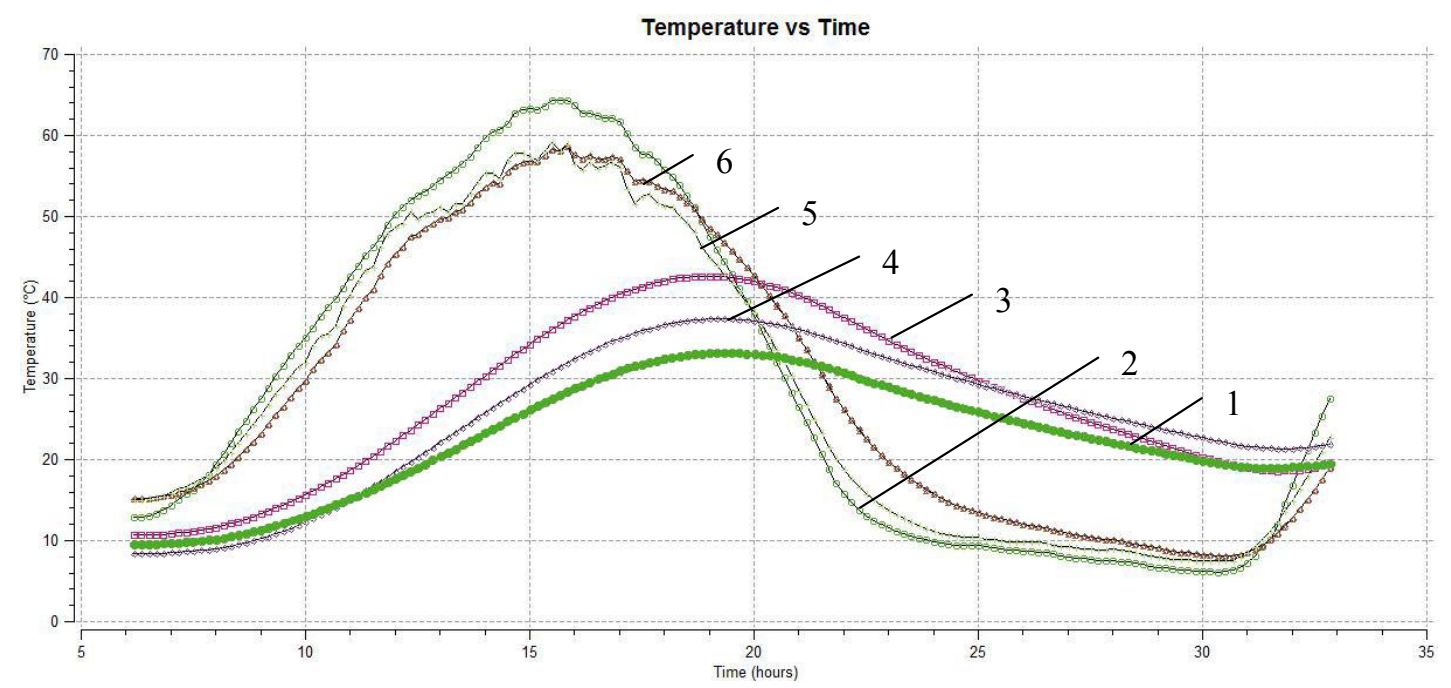

Рис. 3. Графики изменения термодинамических температур: 1 - исследуемый объект; 2 - плита из пенопласта; 3 - стальной лист; 4 - бетонная плита; 5 - деревянная плита (сосна); 6 - плита из красного полнотелого кирпича 

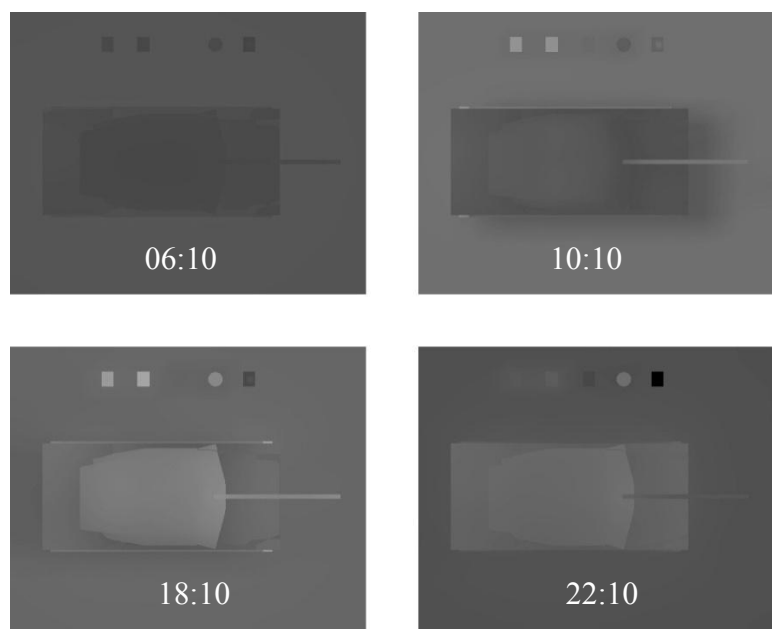

a)
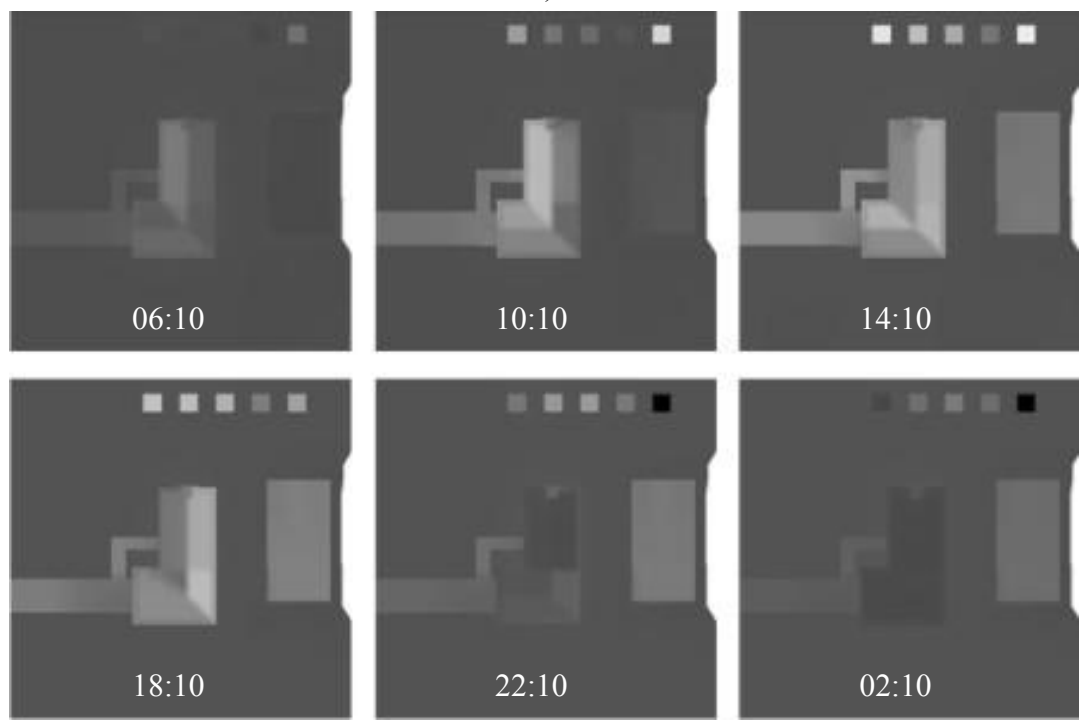

б)

Рис. 4. ИК-сигнатуры техногенных объектов: а - объект техники; б - здание и бункер

Шаг 4. Выбор оптимального по критерию Неймана - Пирсона порогового значения невязки $H_{\mathrm{J}}$ для проведения сегментации кубоида ИК-изображений по заданным эталонным материалам.

Шаг 5. Построение сегментированного изображения в цветовой модели HLS.

В результате применения разработанного алгоритма корреляционной обработки кубоида ИК-изображений были получены сегментированные изображения техногенных объектов, представленные на рис. 5. Анализ полученных тепловых томограмм показывает, что предложенный корреляционный алгоритм позволяет выделить в пределах растра ИК-изображения элементы конструкционных и функциональных материалов, из которых состоит техногенный объект, по сходству их теплофизических параметров эталонным материалам. Так, на рис. 5 


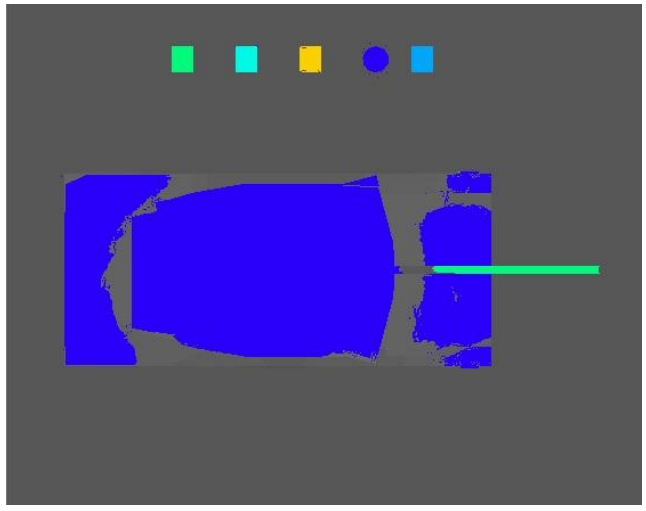

a)

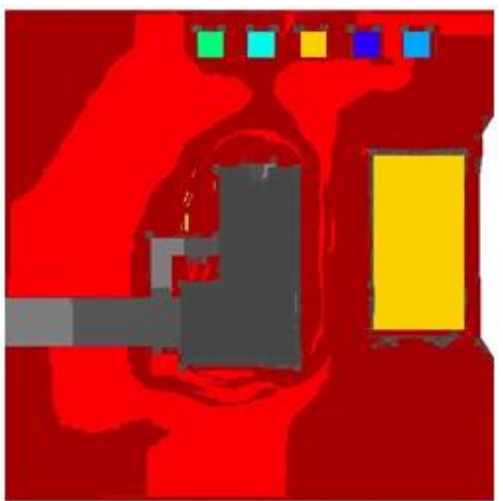

б)

Рис. 5. Сегментированные изображения техногенных объектов: $a$ - объект техники; $\sigma$ - дом и бункер

на фоне местных предметов отчетливо выделен объект «техника», корпус которого отнесен к материалу «стальной лист», а малозаметный объект «бункер» отнесен к материалу «бетонная плита», несмотря на то что он покрыт слоем грунта.

\section{Выводы}

Таким образом, в статье представлен алгоритм корреляционной обработки кубоида ИКизображений в задачах автоматического обнаружения объектов по их теплофизическим параметрам на примере сегментации изображений техногенных объектов, полученных в ходе численного моделирования динамики изменения термодинамических температур на поверхностях исследуемых и эталонных материалов. Для оценки качества работы алгоритма проводилась попиксельная обработка ИК-сигнатур объектов с вычислением невязки и выбором по критерию Неймана - Пирсона пороговых значений невязок, обеспечивающих сегментацию изображений по заданным эталонным материалам. Представленные результаты показали преимущество корреляционной обработки по сравнению с подходом, описанным в работе [9].

Работа выполнена при финансовой поддержке РФФИ (грант № 15-08-02611 А).

\section{Список литературы}

[1] Ищук И.Н., Парфирьев А.В. Измерительная техника, 2014, 10(56), 1162-1166 [Ishchuk, I.N., Parfiriev, A.V. Measurement Techniques, 2014, 10(56), 1162-1166 (in Russian)]

[2] Ищук И.Н., Парфирьев А.В. Измерительная техника, 2014, 1(57), $74-78$ [Ishchuk, I.N., Parfiriev, A.V. Measurement Techniques, 2014, 1(57), $74-78$ (in Russian)]

[3] Yong Xu, Xiangyu Wei, Ge Wang. International Journal of Biomedical Imaging, 2009, Special section, 1-4.

[4] El Hazzat, Soulaiman Saaidi, Abderrahim Karam, Antoine Satori, Khalid. 3D Research. 2015, $6,1-18$.

[5] Ishchuk, I.N., Filimonov, A.M., Tyapkin V.N., Semenov M.E., Kabulova E.G. Modern applied science, 2015, 2, 206-215. 
[6] Ishchuk, I.N., Parfiriev, A.V, Tyapkin V.N., Semenov M.E., Kabulova E.G. Modern applied science, 2015, 3, 233-241.

[7] Khutorskoi M.D., Podgornykh L.V. Reports on Earth Sciences, 2006, 2, 225-229.

[8] Buchnev A.A., Tyapkin V.P. Journal of Siberian Federal University. Engineering \& Technologies, 2015, 6(8), 701-705.

[9] Громов Ю.Ю., Губсков Ю.А., Ищук И.Н., Ворсин И.В. Промышленные АСУ и контроллеры, 2014, 6, 24-28 [Gromov Yu.Yu., Gubskov Yu.A., Ishchuk I.N., Vorsin I.V. Industrial automated control system and controllers, 2014, 6, 24-28 (in Russian)] 